\title{
Prescription Analysis of short dosage forms of metformin as monotherapy or combined therapy in type II diabetes mellitus; pilot study.
}

\author{
Thotawatte N. T ${ }^{\# 1}$, De Silva H. H. V. K. N"2, Rajapaksha R. G. W. D. B ${ }^{\# 3}$ \\ \#Department of Pharmaceutical and Cosmetic Sciences, Faculty of Health Sciences, \\ CINEC Campus, Malabe \\ ${ }^{1}$ nethmithotawatte@gmail.com ${ }^{2}$ Vishwa.DeSilva@cinec.edu ${ }^{3}$ weranga.rajapaksha@cinec.edu
}

\begin{abstract}
This pilot study was done to evaluate the prescribing pattern of insulin and metformin in both monotherapy and combined therapy used in type II diabetes mellitus in Sri Lanka. The study was conducted at community pharmacies and the data was collected for a week. Results shows that total of 67 prescriptions were male $59.7 \%$ and $40.2 \%$ were female. Individuals of the 50-60 year age category was the most risk group having DM and were prescribed with twice a daily, thrice a daily doses of metformin. Different dosage forms were used but the monotherapy was the more frequent prescription in type II DM. The study specifically signified the importance of using the slow releasing dosage form of metformin instead of double dose.
\end{abstract}

Keywords - diabetes mellitus, monotherapy, metformin, insulin, combined therapy

\section{INTRODUCTION}

A prescription is an important document of instruction between a physician and pharmacist in order to prescribe medicines. Prescriptions must be reviewed constantly to prevent irrational prescribing and wastage of medicines [1]. Diabetes mellitus (DM) is a chronic metabolic disease condition where the high glucose level in the blood that is not properly regulated by insulin [2]. DM is listed as one of the majornon-communicable diseases in the world. DM accounts for a vast proportion of morbidity and morta lity across all age groups globally. DM has categorized into two types; Type I and Type II. In Type I DM, the body doesn't produce enough insulin and Type II DM, the body cells do not respond to insulin effectively or insulin resistant [3]. $90 \%$ of diabetes mellitus is identified as Type II DM and related to both insulin resistance and insulin deficiency [4]. A previous study conducted by Wade et al., (1973) in revealed that $20 \%$ of general practitioners prescribed metformin as the oral antidiabetic drug for the treatment for Type II DM and 1343 prescriptions were identified with the insulin [5]. According to the research done by Lee \& Morley, Metformin is the typical oral anti-diabetic drug that is commonly prescribed to Type II diabetic patients [6]. Even though insulin is often used in the treatment of Type I DM and it is also commonly used in combination therapy in Type II DM [3]. A previous research study was done by Yki-Jarvinen, concluded that the dose of insulin in combination therapy is lower compared to the administration of insulin monotherapy. The purpose of this observational study was to statistically analyse qualitatively and quantitatively monotherapy or combined therapy in Type II DM..

\section{MATERIALS AND METHODS}

This study was conducted by analysing the drug prescriptions to evaluate the antidiabetic prescribing pattems in Sri Lanka. The prescriptions were collected with consent of the patient from random community pharmacies for a week on alternate days and categorized according to the inclusion and exclusion criteria. The following data were summarized in a designed data entry form using collected prescriptions including antihyperglycemic medicines (insulin, metformin or both), the dosage of the medication prescribed and gender of the patient and age of the patient. Data a nalysis was performed using IBM SPSS 21.00 statistical package for windows.

\section{RESULTS}

Among the collected prescription, 67 prescriptions were selected for evaluation. According to the data collected, 40 $(59.7 \%)$ were males and $27(40.2 \%)$ were females. Most of the collected prescriptions were in the 50-60 years age category (Table 1).

According to the numerals presented in the above table the $50-60$ year age gap is most at risk and most a ffected by type II $\mathrm{DM}$

The data were further analysed to determine the count of patients prescribed with metformin, insulin and combined therapy. According to the analysed prescription data, 58 from selected 67 prescriptions were prescribed with metformin only and the remaining 9 prescriptions were prescribed with combined therapy of insulin and metformin. The results revealed that no patients were prescribed with insulin only a mong the a lready collected prescriptions. (Fig.1).

TABLE I

DEMOGRAPHIC DATA EVALUATED IN THE PRESCRIPTIONS

\begin{tabular}{l|l}
\hline \multicolumn{2}{c|}{ Demographic data } \\
\hline Sex & (40) $59.7 \%$ \\
Male & (27) $40.2 \%$ \\
Female & \\
Age group in years & \\
$20-30$ & (1) $1.5 \%$ \\
$31-40$ & (4) $6.2 \%$ \\
$41-50$ & (16) $24.6 \%$ \\
$51-60$ & (29) $44.6 \%$ \\
Above 60 & (15) $23.1 \%$ \\
\hline
\end{tabular}




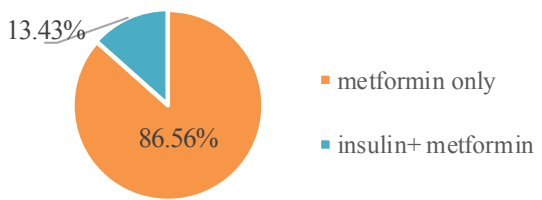

Fig 1 Percentage of prescribed medications; the metformin only medication was prescribed to a majority of patients in 58 prescriptions while only 9 prescriptions were prescribed with both insulin and metformin (combined therapy).

Analysing the collected data on several doses of metformin prescribed by the physicians were found that, the most common dose was $500 \mathrm{mg}$ of metform in to be taken 2 times per day (bd) across all age groups (Fig.2). This accounted for a significant $61.8 \%$. In some instances, the patients were advised to take $500 \mathrm{mg}$ of metform in tablets 3 times perday (tds) which is significant $10.9 \%$.

According to the prescribing pattern of insulin, patients have administered 30 units in the morning and 20 units at night which was conveniently denoted a s $30 / 20$ units. However, due to non-existent of data for prescriptions with 'insulin only' medication, the data for combined therapy of 30/20 units of insulin combined with $500 \mathrm{mg}$ metformin was analysed. Statistical analysis demonstrated that combined therapy for type II DM was greatly practised for the patients with 40-50 yearage category.

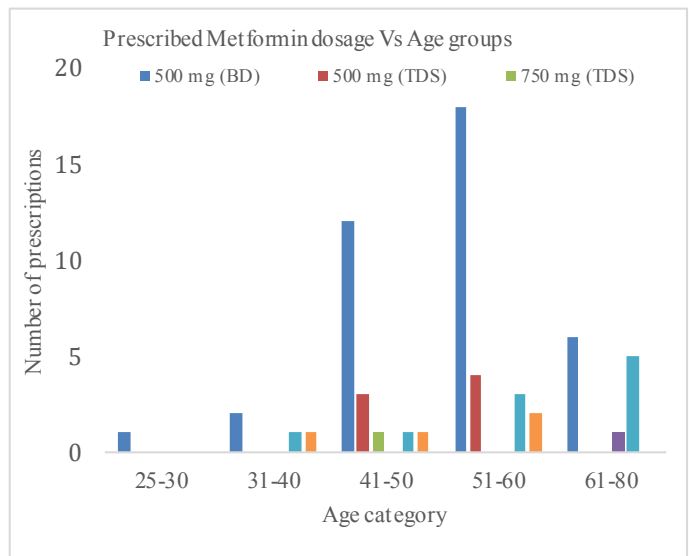

Fig 2 The abundance of metformin dosages in different age groups; analysis of metformin only prescriptions portrayed that $500 \mathrm{mg}(\mathrm{bd})$ was the most common dose of metformin prescribed across all age categories and the above dose was most prescribed in the 50-60 year age category.

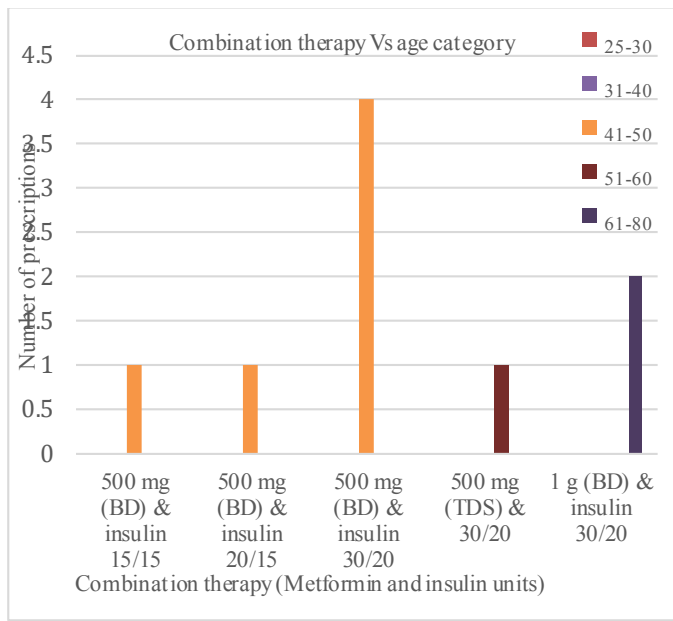

Fig 3 Abundance of combined therapy dosages in different age groups: analysis of 9 prescriptions of combined therapy portrayed that $30 / 20$ units of insulin was the most common insulin dose and also combined therapy was seen to be prescribed more in the 41-50 year category.

The results show that most common drug in use for the type II DM was metformin $(87 \%)$ and there was less variation in the dosages in which metformin was prescribed in both combined and monotherapy.

\section{IV.DISCUSSION}

Our study revealed that males were affected or at high risk of having type II DM when compared to females. Our results is compatible with the findings of Mahmood et al., (2017) males were at high risk for type II DM due to the social habits and lifestyle changes in men [4]. Previous studies have revealed that macrovascular complications including ischaemic heart disease and stroke are the main causes of morbidity and mortality among DM patients. This risk is observed to be higher among the men who regularly smoke. [8]. They have difficulties in regulation, dosing of insulin and controlling their disease compared to non-smokers. The use of tobacco can increase blood glucose and IR as previously reported. Apart from that, consumption of alcohol has some adverse effects on DM like chronic pancreatic inflammation which can hinder its ability to secrete insulin [4].

This observational study also showed that the 50-60 years group was mostly prescribed for treatment with type II DM. According to the Mahmood et al., (2017) and co-worker's study, the age category 40-60 were most at risk for developing type II DM, who are the most committed and productive of their lives due to increase in work-related stress and physical body changes [4].

Metformin is the drug of choice for type II DM prescribed with $500 \mathrm{mg}$ tablets to be taken as 2 times per day, as observed in our study. In combined therapy, the same amount of metformin was coupled with 30/20 units of insulin. Most 
diabetic patients were prescribed with different short dosage forms of metformin as monotherapy as well as of combined therapy. In chronic disea ses, continuous lifelong treatment is a mandatory strategy and most of the research findings had shown that patients experience poor drug adherence and compliance with minimum control over the disease progression. This is compatible with our research findings of multiple therapy with short acting drug formulae for DM. With this pilot study, we suggest for medical professionals to re consider of substitution of long acting drugs instea d of short acting formulations. This study can extend to evaluate the patients drug a dherence and compliance on short dosa ge forms vs slow releasing formulae.

\section{CONCLUSION}

Our finding in this small scale study indicated that preponderance of male gender for type II DM through prescription via cross sectional survey. Most importantly there is obvious evidence for high frequent usage of for short dosage form of double dose $(500 \mathrm{mg}$ bd) of metformin in the form of both monotherapy and in combined therapy with insulin. There is a significant number of other frequent dosage forms and we could nto find any single use of slow releasing formulae. This is not suitable for the DM patients of age population to reduce poor drug compliance.

Therefore we decided to continue our study to research on patients drug compliance and drug adherence on therapeutic efficacy of DM.

\section{REFERENCES}

[1] M. H. Sumana and Santoshkumar A Shetti, "Prescription Analysis of drugs used in outpatient department of dermatology at tertiary care hospital", Asian Journal of Biomedical and Pharmaceutical Sciences, vol.5, pp. 2224, July 2015.

[2] Mayo Clinic, Type 2 diabetes - Symptoms and causes, 2019. [online] Available at: https://www.mayoclinic.org/diseases-conditions/type-2diabetes/symptoms-causes/syc-20351 193 [Accessed 12 Dec. 2019].

[3] K. Cherney, List of Common Diabetes Medications, 2019. [online] Health line. Available at: https://www.healthline.com/health/diabetes/medications-list [Accessed 12 Dec. 2019].

[4] M. Mahmood, R.C. Reddy et al., "Prescription Analysis of anti-diabetic drugs in diabetes mellitus and associated comorbidities", Clinical Investigation, vol.8, pp.5-12, Nov 2017.

[5] O.L.Wade, D.R.Hadden, and H. Hood, "The prescribing of drugs used in the treatment of diabetes." British journal of preventive \& social medicine, vol.27(1), pp. 44-48, 1973. doi: 10.1136/jech.27.1.44.

[6] A. Lee, and J.E. Morley, "Metformin decreases food consumption and induces weight loss in subjects with obesity with type II non-insulindependent diabetes", Obesity Research, vol.6(1), pp. 47-53, 1998. doi: 10.1002/j.1550-8528.1998.tb00314.x.

[7] H. Yki-Järvinen, (2001) "Combination therapies with insulin in type 2 diabetes", Diabetes Care, vol.24(4), pp. 758-767, 2001. doi: 10.2337/diacare.24.4.758.

[8] D. Campagna, A. Alamo, A. Di Pino et al., "Smoking and diabetes: dangerous liaisons and confusing relationships", Diabetol Metab Syndr, vol.11, pp. 85,2019. doi.org/10.1186/s 13098-019-0482-2

October 2020 